Sādhanā Vol. 40, Part 1, February 2015, pp. 215-234. (C) Indian Academy of Sciences

\title{
A simplified four-unknown shear and normal deformations theory for bidirectional laminated plates
}

\author{
A M ZENKOUR \\ Department of Mathematics, Faculty of Science, King Abdulaziz University, \\ P.O. Box 80203, Jeddah 21589, Saudi Arabia \\ Department of Mathematics, Faculty of Science, Kafrelsheikh University, \\ Kafr El-Sheikh 33516, Egypt \\ e-mail: zenkour@gmail.com; zenkour@kau.edu.sa
}

MS received 30 October 2012; revised 21 June 2014; accepted 20 September 2014

\begin{abstract}
This paper presents a simplified 4-unknown shear and normal deformations theory for the bending analysis of cross-ply laminated plates. The present theory accounts for an adequate distribution of transverse shear strains through the plate thickness and tangential stress-free on the plate surfaces. The effect of normal strain is also included. The governing, equilibrium equations and boundary conditions are derived by employing the virtual work principle. Numerical results for stresses and displacements are compared well with those obtained using 3-D elasticity solution.
\end{abstract}

Keywords. Cross-ply laminates; four-unknown sinusoidal plate theory.

\section{Introduction}

The modifications of plate theories are still the most interesting topics in the literature. Most of these theories are concerned with the so called equivalent single-layer plate theory. It contains, for example, the classical (CPT), first-order (FPT), higher-order (HPT), and other shear deformation theories. These theories have been applied to a variety of mechanical problems related to composite plates. The CPT ignores the transverse shear deformation and gives reliable results only for thin structures. The FPT is simple to implement and applied for both moderately thick and thin laminated plates and gives acceptable results but depends on a shear correction factor which is hard to find as it depends on many parameters. However, there is no need of shear correction factors when using HPT or other shear deformation plate theories but governing equations are more complicated than those of the FSDT. In fact, the HPT and other refined shear deformation theories give more accurate and stable transverse shear stresses.

In this article, a simplified shear and normal deformations plate theory is presented. Just four unknown displacement functions are used. The effects due to transverse shear and normal deformations are both included. The effectiveness of the present theory is demonstrated 
through illustrative problem and results are compared with the corresponding 3-D elasticity solution.

\section{A simplified four-unknown plate theory}

To describe various plate theories, we introduce the following coordinate system. The $x, y$ and $z$ coordinates are taken along the length, the width, and the thickness of the plate, respectively. All applied mechanical loads and geometry are such that the displacements $u_{i}$ along the coordinate system are functions of $x, y, z$ and time $t$. With respect to the equivalent single-layer theories, the displacements for static problems are expanded in terms of various powers of the thickness coordinate $z$ and unknown functions of $x$ and $y$ :

$$
U_{i}(x, y, z)=\sum_{j=0}^{\infty} z^{j} u_{i}^{(j)}(x, y), \quad i=1,2,3,
$$

where $u_{i}^{(j)}(x, y)$ are undetermined functions. The displacements $u_{i}$ of a material point in the plate are assumed with the aid of a refined shear deformation plate theory (RPT) as (Zenkour 2004a, b, 2005a, b, 2006, 2007a)

$$
\left.\begin{array}{c}
u_{1}=u(x, y)-z \frac{\partial w}{\partial x}+f(z) \psi_{1}(x, y), \\
u_{2}=v(x, y)-z \frac{\partial w}{\partial y}+f(z) \psi_{2}(x, y), \\
u_{3}=w(x, y)+f_{\prime}(z) \varphi(x, y),
\end{array}\right\}
$$

where $u, v$ and $w$ are the displacements of the middle surface along the axes $x, y$ and $z$, respectively, $\psi_{1}$ and $\psi_{2}$ are the rotations about the $y$ and $x$ axes and account for the effect of transverse shear and $\varphi$ is an additional displacement account for the effect of normal stress. The coefficient of $\psi_{1}$ and $\psi_{2}$ is given by $f$ and it should be odded to function of $z$. The prime denotes differentiation with respect to $z$. The displacement field of classical and shear deformation theories are given by taking suitable forms for $f(z)$ :

Classical plate theory (CPT) (Love 1944): $f(z)=0$,

First-order plate theory (FPT) (Mindlin 1951; Reissner 1944, 1945): $f(z)=z$ and $\varphi=0$, Higher-order plate theory (HPT) (Reddy 1984): $f(z)=z\left[1-\frac{4}{4}\left(\frac{z}{h}\right)^{2}\right]$ and $\varphi=0$,

Simple sinusoidal plate theory (SPT) (Zenkour 2004a, b; 2005a, b; 2006): $f(z)=\frac{h}{\pi} \sin \left(\frac{\pi z}{h}\right)$ and $\varphi=0$.

The displacement field given in Eq. (2) with $\varphi=0$ was presented by Touratier (1991) and extended to functionally graded plates by Zenkour (2006). In recent years, many investigators have used the same displacement field with the same or different forms of the function $f(z)$. Ferreira et al (2005) have used a trigonometric shear deformation theory for modelling symmetric composite plates discretized by a meshless method based on global multi-quadric radial basis functions. Khabbaz et al (2009) have used the energy concept along with the first- and third-order shear deformation theories based upon Eq. (2) to predict the large deflection and through the thickness stress of FGM plates. Aydogdu (2009) has proposed a new higher-order shear deformable laminated composite plate theory from the 3-D elasticity bending solutions by 
using an inverse method. Jun \& Hongxing (2009) have developed the exact dynamic stiffness matrix of a uniform laminated composite beam based on trigonometric shear deformation theory. Xiang et al (2009) have used various shear deformation theories for modelling isotropic, sandwich and laminated plates. A generalized higher-order theory describing the mechanical behaviour of multi-layered composite plates with arbitrary lamination scheme was proposed by Fiedler et al (2010). Batista (2010) has derived the equations of a moderately thick plate by the method of successive approximations. Ferreira \& Roque (2011) have used a higher-order shear deformation theory and a radial basis function collocation technique for predicting the static deformations and free vibration behaviour of thick plates. Neves et al (2012a, b) have presented an original hyperbolic sine shear deformation theory for the bending and free vibration analysis of functionally graded plates. Mantari et al (2012a, b) have presented an analytical solution to the static analysis of functionally graded plates and sandwich and composite plates, using a recently developed higher-order shear deformation theory. Additional 3-D elasticity theory may be found in the literature (see, for instance, Pagano (1970), Pagano \& Hatfield (1972), Kant et al (2008) and Kapuria \& Kumari (2010)).

\section{Basic equations}

Consider a composite rectangular plate of length $a$, width $b$ and thickness $h$ made of an arbitrary lamination material (see figure 1). The plate is subjected to a transverse distributed load $q(x, y)$. The plate is referred to as coordinate system $(x, y, z)$ with the co-ordinates and $y$ along the inplane directions and $z$ along the thickness direction. The plate composed of orthotropic layers oriented at angles $\theta_{1}, \theta_{2}, \ldots, \theta_{n}$. The material of each layer is assumed to posses one plane of elastic symmetry parallel to the $x-y$ plane. Perfect bonding between the orthotropic layers and temperature-independent mechanical and thermal properties may be assumed.

The refined shear deformation plate theory that includes the effect of both transverse shear and normal deformations was first given by Zenkour (2007a). It is based upon the displacements $u_{i}$ with $\varphi \neq 0$ as mentioned in Eq. (2). It contains only one additional dependent unknown of other shear deformation theories, but takes into accounts of the normal deformation through the thickness of the plate using a sine-law distribution. No transverse shear correction factors are needed for the RPT, as well as HPT and SPT, because a correct representation of the transverse

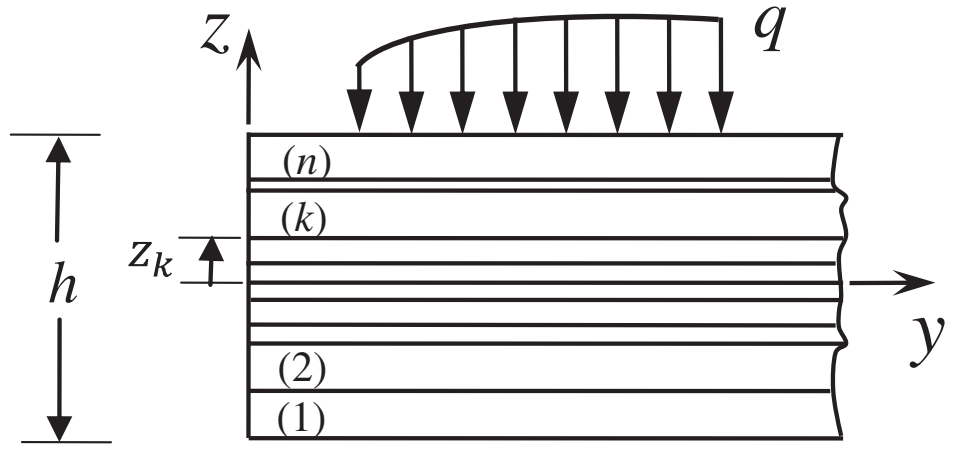

Figure 1. Schematic diagram for the laminated plate. 
shearing strain is given. We noticed that there is an interesting relation between the unknown functions $\varphi$ in one side and $\psi_{1}$ and $\psi_{2}$ the other side. This relation may be summarized by

$$
\psi_{1}(x, y)=\alpha_{1} \frac{\partial \varphi}{\partial x}, \quad \psi_{2}(x, y)=\alpha_{2} \frac{\partial \varphi}{\partial y},
$$

where $\alpha_{1}$ and $\alpha_{2}$ are arbitrary parameters that may be given in terms of the plate dimensions. So, the refined four-unknown shear deformation plate theory is given by

$$
\left.\begin{array}{c}
u_{1}=u(x, y)-z \frac{\partial w}{\partial x}+\alpha_{1} f(z) \frac{\partial \varphi}{\partial x}, \\
u_{2}=v(x, y)-z \frac{\partial w}{\partial y}+\alpha_{2} f(z) \frac{\partial \varphi}{\partial y}, \\
u_{3}=w(x, y)+f_{\prime}(z) \varphi(x, y)
\end{array}\right\} .
$$

The above displacement field contains only four unknown functions $u, v, w$, and $\varphi$. In addition, the effects due to transverse shear strain and normal deformation are both included.

The stress-strain relations for a linear elastic plate for the $k$ th layer are given by treating each layer as an individual homogeneous plate and they are written in the form:

$$
\left\{\begin{array}{l}
\sigma_{x} \\
\sigma_{y} \\
\sigma_{z}
\end{array}\right\}^{k}=\left[\begin{array}{lll}
c_{11} & c_{12} & c_{13} \\
c_{12} & c_{22} & c_{23} \\
c_{13} & c_{23} & c_{33}
\end{array}\right]^{k}\left\{\begin{array}{l}
\varepsilon_{x} \\
\varepsilon_{y} \\
\varepsilon_{z}
\end{array}\right\},
$$

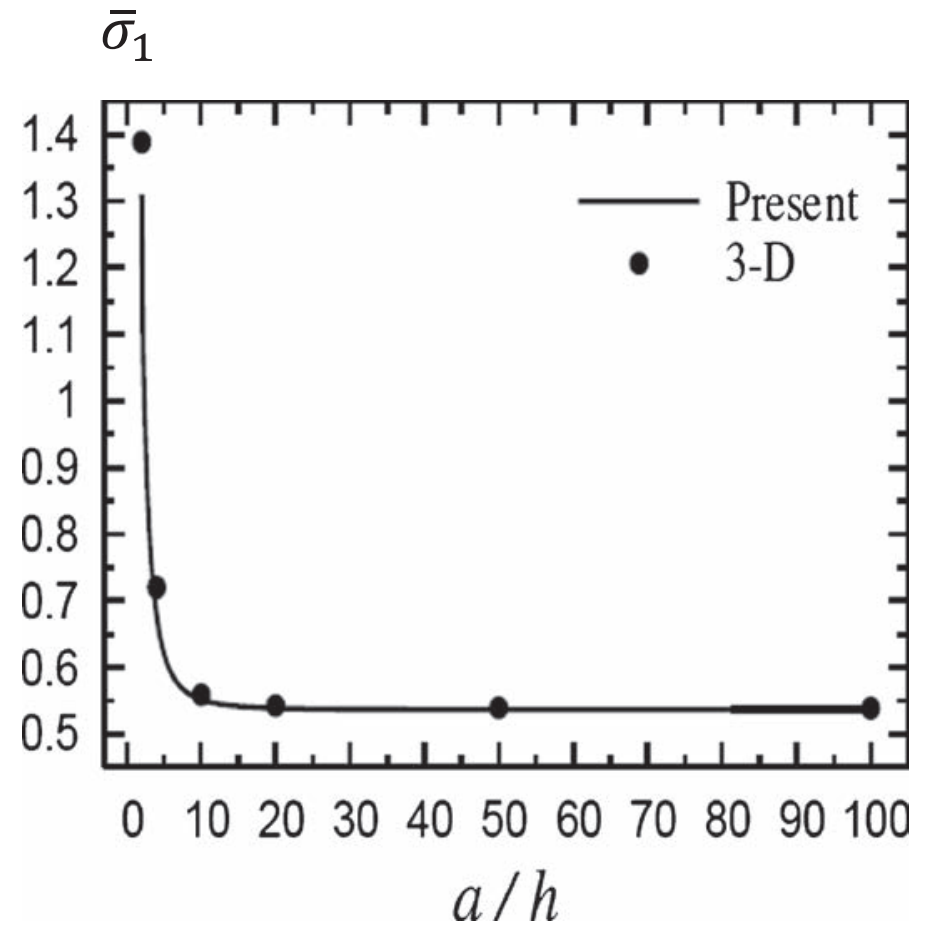

Figure 2. The in-plane longitudinal stress $\bar{\sigma}_{1}$ versus the side-to-thickness ratio $a / h$ of a $\left(0^{\circ} / 90^{\circ} / 90^{\circ} / 0^{\circ}\right)$ square plate. 


$$
\tau_{y z}^{(k)}=c_{44}^{(k)} \gamma_{y z}, \quad \tau_{x z}^{(k)}=c_{55}^{(k)} \gamma_{x z}, \quad \tau_{x y}^{(k)}=c_{66}^{(k)} \gamma_{x y},
$$

where $c_{i j}^{(k)}$ are the transformed elastic coefficients. The strain components $\varepsilon_{i}$ and $\gamma_{i j}$ are expressed in terms of the displacement quantities as:

$$
\begin{gathered}
\left\{\begin{array}{c}
\varepsilon_{x} \\
\varepsilon_{y} \\
\gamma_{x z}
\end{array}\right\}=\left\{\begin{array}{c}
\varepsilon_{x}^{0} \\
\varepsilon_{y}^{0} \\
\gamma_{x y}^{0}
\end{array}\right\}+z\left\{\begin{array}{c}
\kappa_{x} \\
\kappa_{y} \\
\kappa_{x y}
\end{array}\right\}+f(z)\left\{\begin{array}{c}
\eta_{x} \\
\eta_{y} \\
\eta_{x y}
\end{array}\right\}, \\
\varepsilon_{z}=f^{\prime \prime}(z) \varepsilon_{z}^{0}, \quad \gamma_{x z}=f^{\prime}(z) \gamma_{x z}^{0}, \quad \gamma_{y z}=f^{\prime}(z) \gamma_{y z}^{0},
\end{gathered}
$$

where

$$
\begin{gathered}
\varepsilon_{x}^{0}=\frac{\partial u}{\partial x}, \quad \varepsilon_{y}^{0}=\frac{\partial v}{\partial y}, \quad \gamma_{x y}^{0}=\frac{\partial v}{\partial x}+\frac{\partial u}{\partial y}, \quad \varepsilon_{z}^{0}=\varphi, \quad \gamma_{y z}^{0}=\left(1+\alpha_{2}\right) \frac{\partial \varphi}{\partial y}, \\
\gamma_{x z}^{0}=\left(1+\alpha_{1}\right) \frac{\partial \varphi}{\partial x}, \quad \kappa_{x}=-\frac{\partial^{2} w}{\partial x^{2}}, \quad \kappa_{y}=-\frac{\partial^{2} w}{\partial y^{2}}, \quad \kappa_{x y}=-2 \frac{\partial^{2} w}{\partial x \partial y} \\
\eta_{x}=\alpha_{1} \frac{\partial^{2} \varphi}{\partial x^{2}}, \quad \eta_{y}=\alpha_{2} \frac{\partial^{2} \varphi}{\partial y^{2}}, \quad \eta_{x y}=\left(\alpha_{1}+\alpha_{2}\right) \frac{\partial^{2} \varphi}{\partial x \partial y} .
\end{gathered}
$$

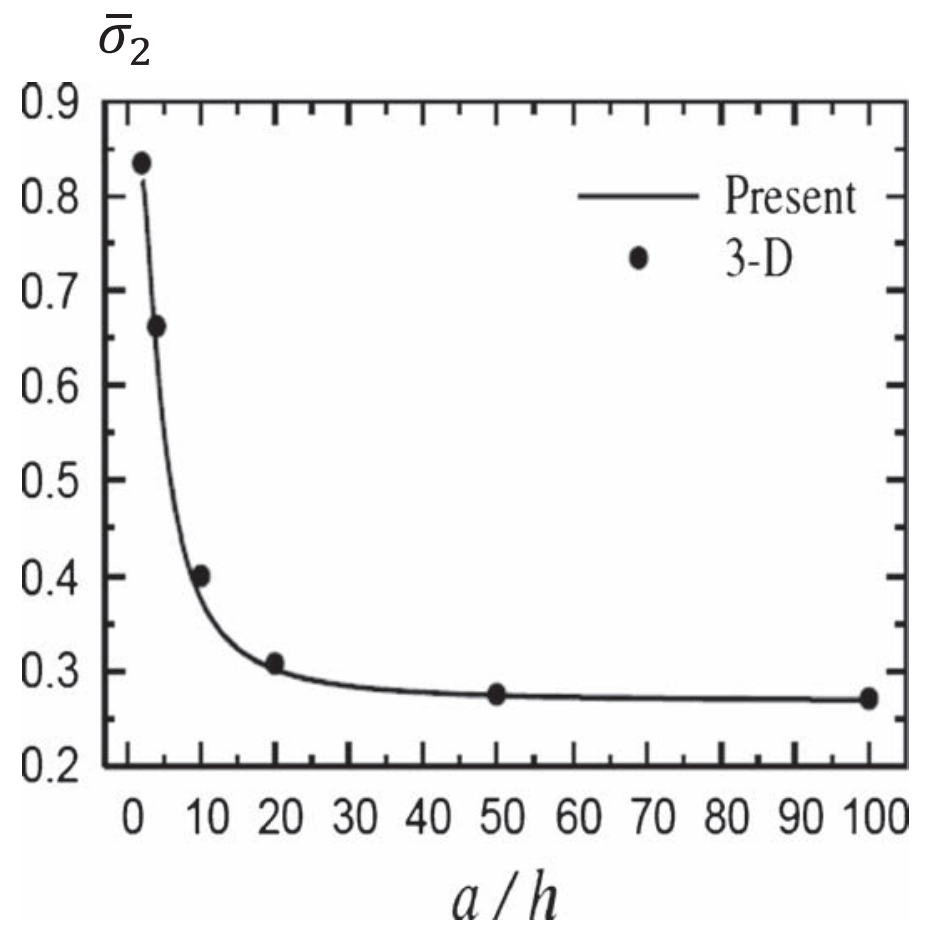

Figure 3. The in-plane normal stress $\bar{\sigma}_{2}$ versus the side-to-thickness ratio $a / h$ of a $\left(0^{\circ} / 90^{\circ} / 90^{\circ} / 0^{\circ}\right)$ square plate. 
The stress, moment, and additional moment resultants of a laminated composite plate made up of $n$ layers of orthotropic laminas can be obtained by integrating Eq. (5) over the thickness, and are written as

$$
\begin{gathered}
\left\{\begin{array}{c}
N_{x} \\
N_{y} \\
M_{x} \\
M_{y} \\
S_{x} \\
S_{y} \\
S_{z}
\end{array}\right\}=\left[\begin{array}{lllllll}
A_{11} & A_{12} & B_{11} & B_{12} & B_{11}^{a} & B_{12}^{a} & G_{13}^{a} \\
A_{12} & A_{22} & B_{12} & B_{22} & B_{12}^{a} & B_{22}^{a} & G_{23}^{a} \\
B_{11} & B_{12} & D_{11} & D_{12} & D_{11}^{a} & D_{12}^{a} & H_{13}^{a} \\
B_{12} & B_{22} & D_{12} & D_{22} & D_{12}^{a} & D_{22}^{a} & H_{23}^{a} \\
B_{11}^{a} & B_{12}^{a} & D_{11}^{a} & D_{12}^{a} & F_{11}^{a} & F_{12}^{a} & L_{13}^{a} \\
B_{12}^{a} & B_{22}^{a} & D_{12}^{a} & D_{22}^{a} & F_{12}^{a} & F_{22}^{a} & L_{13}^{a} \\
G_{13}^{a} & G_{23}^{a} & H_{13}^{a} & H_{23}^{a} & L_{13}^{a} & L_{23}^{a} & P_{33}^{a}
\end{array}\right]\left\{\begin{array}{c}
\varepsilon_{x}^{0} \\
\varepsilon_{y}^{0} \\
\kappa_{x} \\
\kappa_{y} \\
\eta_{x} \\
\eta_{y} \\
\varepsilon_{z}^{0}
\end{array}\right\}, \\
\left\{\begin{array}{c}
N_{x y} \\
M_{x y} \\
S_{x y}
\end{array}\right\}=\left[\begin{array}{c}
A_{66} B_{66} B_{66}^{\alpha} \\
B_{66} D_{66} D_{66}^{\alpha} \\
A_{66}^{\alpha} D_{66}^{\alpha} F_{66}^{\alpha}
\end{array}\right]\left\{\begin{array}{l}
\gamma_{x y}^{0} \\
\kappa_{x y} \\
\eta_{x y}
\end{array}\right\}, Q_{y z}=P_{44}^{\alpha} \gamma_{y z}^{0}, Q_{x z}=P_{55}^{\alpha} \gamma_{x z}^{0},
\end{gathered}
$$

$\bar{\sigma}_{6}$

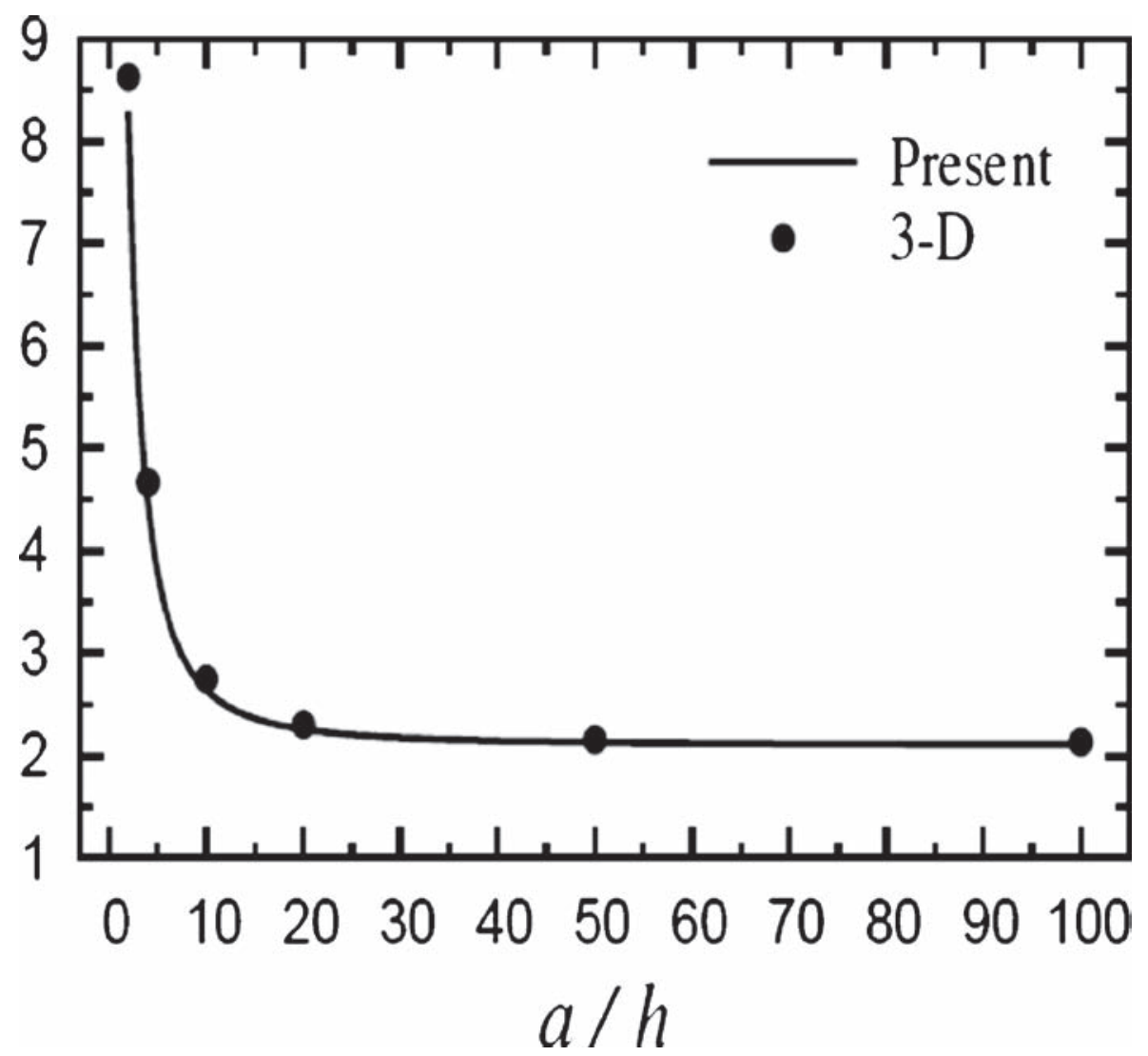

Figure 4. The in-plane tangential stress $\bar{\sigma}_{6}$ versus the side-to-thickness ratio $a / h$ of a $\left(0^{\circ} / 90^{\circ} / 90^{\circ} / 0^{\circ}\right)$ square plate. 
where $A_{i j}$ and $B_{i j} ; \ldots$ etc., are the laminate stiffness coefficients and they are defined in terms of the reduced stiffness coefficients $c_{i j}^{(k)}$ for the layers $k=1,2, \ldots, n$, as:

$$
\begin{gathered}
\left\{A_{i j}, B_{i j}, D_{i j}\right\}=\sum_{k=1}^{n} \int_{z_{k}}^{z_{k+1}} c_{i j}^{(k)}\left\{1, z, z^{2}\right\} \mathrm{d} z, \quad i, j=1,2,6, \\
\left\{B_{i j}^{a}, D_{i j}^{a}, F_{i j}^{a}\right\}=\sum_{k=1}^{n} \int_{z_{k}}^{z_{k+1}} c_{i j}^{(k)} f(z)\{1, z, f(z)\} \mathrm{d} z, \quad i, j=1,2,6, \\
\left\{G_{i 3}^{a}, H_{i 3}^{a}, L_{i 3}^{a}\right\}=\sum_{k=1}^{n} \int_{z_{k}}^{z_{k+1}} c_{i j}^{(k)} f^{\prime \prime}(z)\{1, z, f(z)\} \mathrm{d} z, \quad i=1,2, \\
P_{i i}^{a}=\sum_{k=1}^{n} \int_{z_{k}}^{z_{k+1}} c_{i i}^{(k)}\left[f^{\prime}(z)\right]^{2} \mathrm{~d} z, \quad i=4,5
\end{gathered}
$$

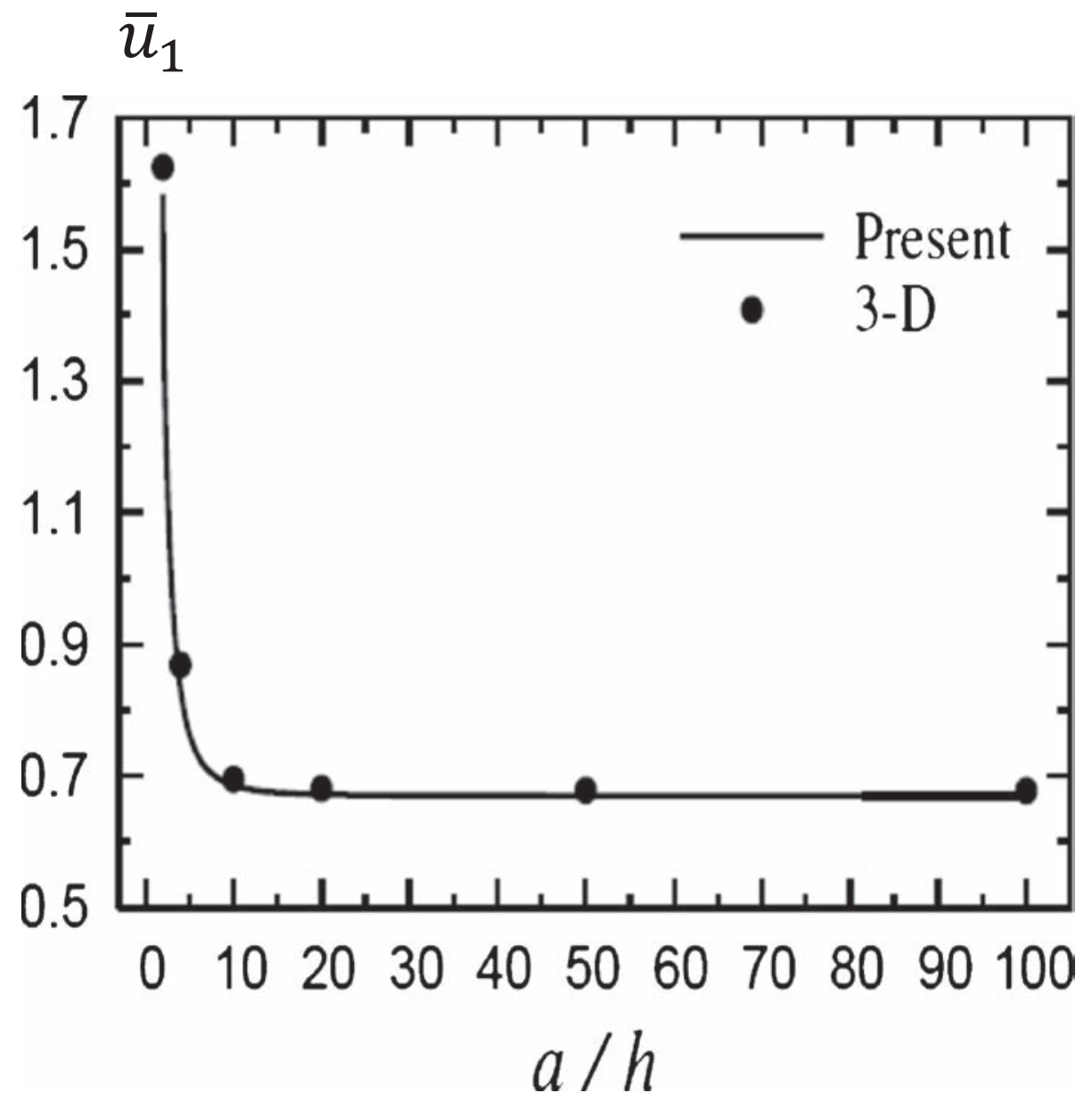

Figure 5. The in-plane displacement $\bar{u}_{1}$ versus the side-to-thickness ratio $a / h$ of a $\left(0^{\circ} / 90^{\circ} / 90^{\circ} / 0^{\circ}\right)$ square plate. 


$$
P_{33}^{a}=\sum_{k=1}^{n} \int_{z_{k}}^{z_{k+1}} c_{33}^{(k)}\left[f^{\prime \prime}(z)\right]^{2} \mathrm{~d} z .
$$

\section{Governing equations}

The governing equations can be carried out using the principle of virtual displacements. First, the equilibrium equations associated with the present theory are

$$
\left.\begin{array}{c}
\frac{\partial N_{x}}{\partial_{x}}+\frac{\partial N_{x y}}{\partial_{y}}=0, \quad \frac{\partial N_{x y}}{\partial x}+\frac{\partial N_{y}}{\partial y}=0, \quad \frac{\partial^{2} M_{x}}{\partial_{x^{2}}}+2 \frac{\partial^{2} M_{x y}}{\partial x \partial y}+\frac{\partial^{2} M_{x y}}{\partial y^{2}}+q=0, \\
S_{z}+\alpha_{1} \frac{\partial^{2} S_{x}}{\partial x^{2}}+\left(\alpha_{1}+\alpha_{2}\right) \frac{\partial^{2} S_{x y}}{\partial x \partial y}+\alpha_{2} \frac{\partial^{2} S_{y}}{\partial y^{2}}-\left(1+\alpha_{1}\right) \frac{\partial Q_{x y}}{\partial x}-\left(1+\alpha_{2}\right) \frac{\partial Q_{y z}}{\partial_{y}}=0 .
\end{array}\right\} .
$$

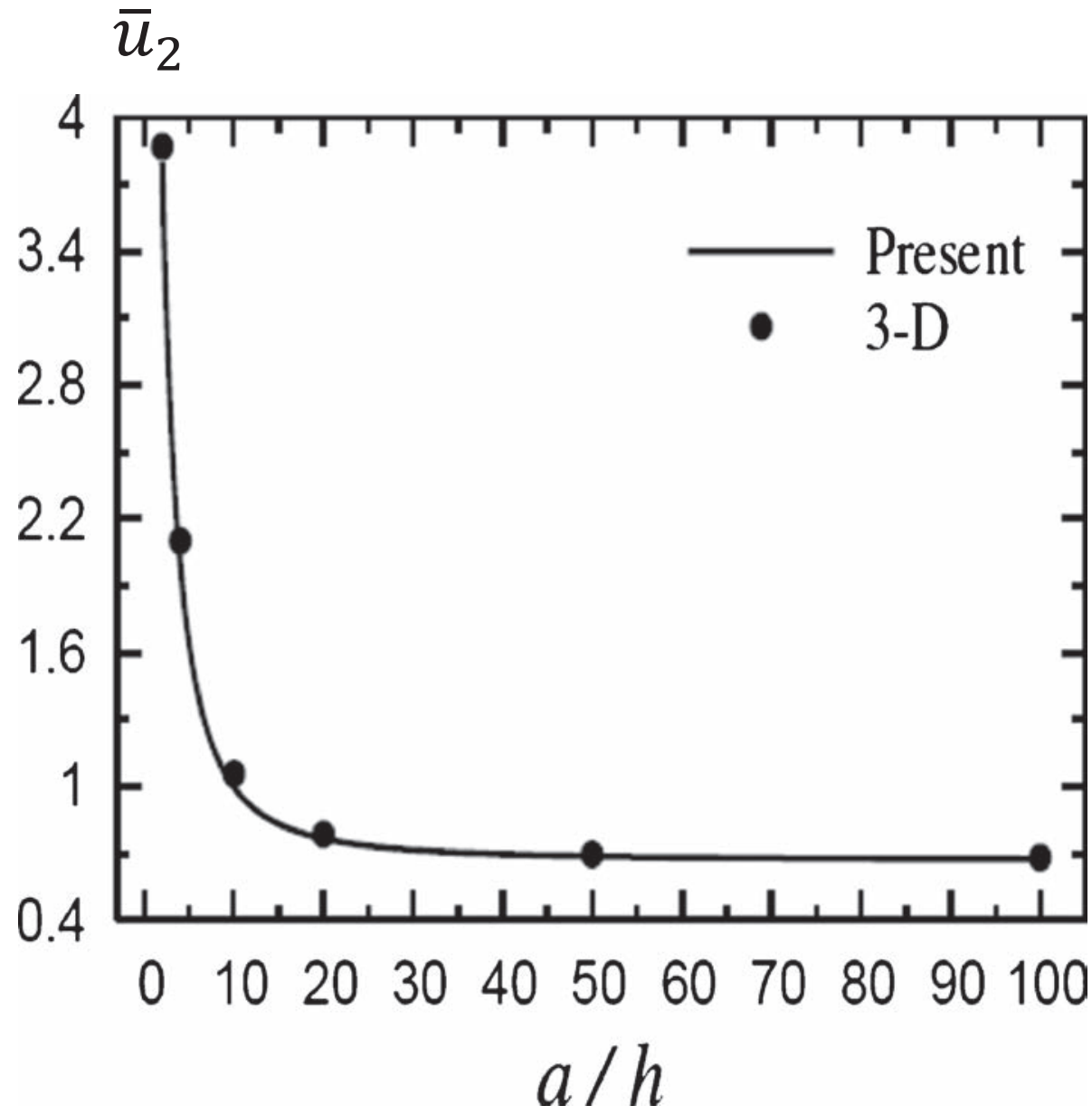

Figure 6. The in-plane displacement $\bar{u}_{2}$ versus the side-to-thickness ratio $a / h$ of a $\left(0^{\circ} / 90^{\circ} / 90^{\circ} / 0^{\circ}\right)$ square plate. 
Substituting the stress resultants given in Eq. (8) into the above equations, one obtains the following operator equation,

$$
[J]\{\delta\}=\{Q\},
$$

where $\{\delta\}=\{u, v, w, \varphi\}^{t}$ is the displacement field, $\{Q\}=\{0,0,-q(x, y), 0\}^{t}$ is the load vector, and $[J]$ is the symmetric matrix of differential operators and its elements are given in Appendix A.

Second, the principle of virtual displacements also gives the requirements of the kinematic and dynamic boundary conditions as follows.

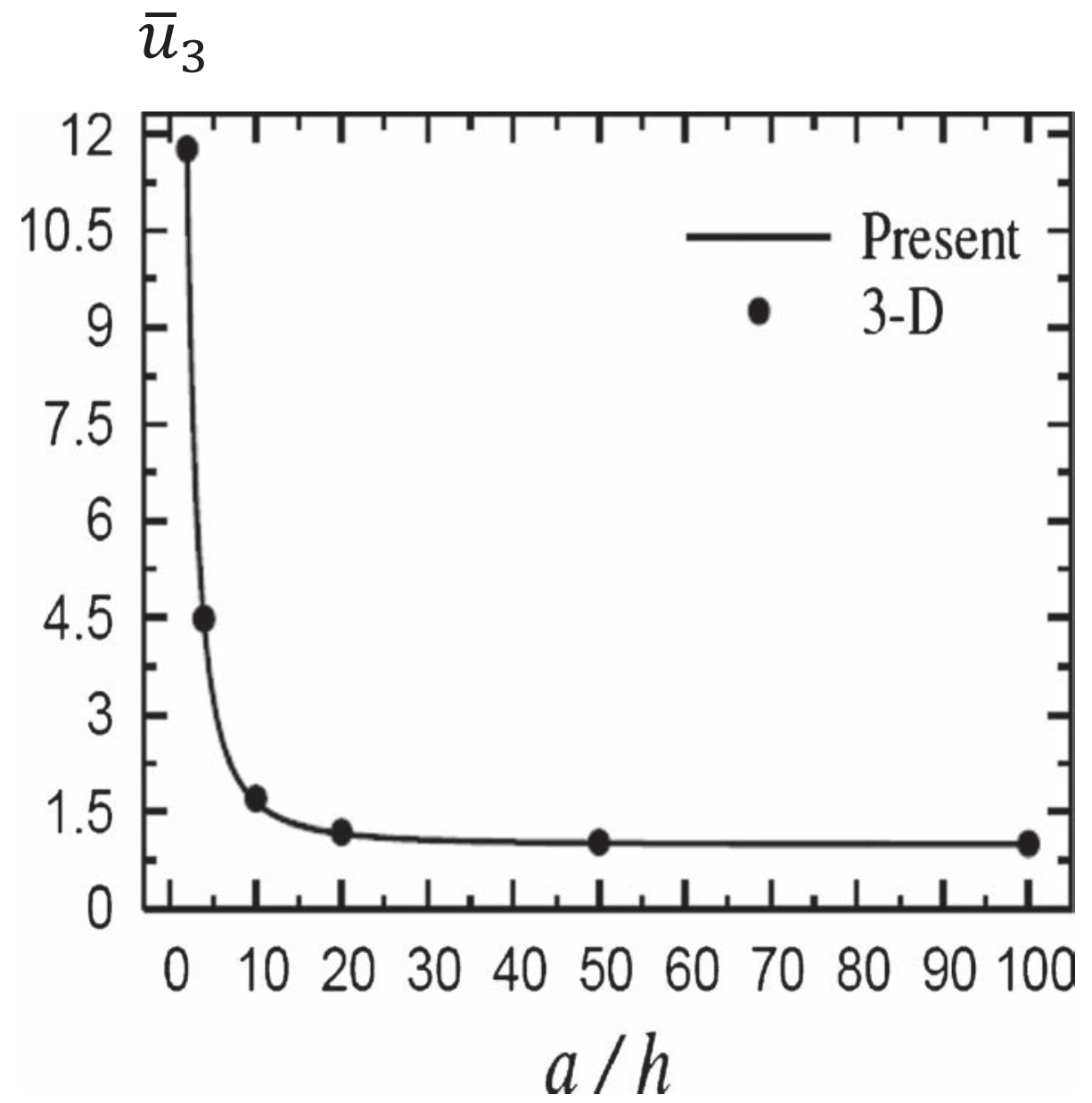

Figure 7. The out-of-plane displacement $\bar{u}_{3}$ versus the side-to-thickness ratio $a / h$ of a $\left(0^{\circ} / 90^{\circ} / 90^{\circ} / 0^{\circ}\right)$ square plate. 
At edge $x=0, a \quad$ At edge $y=0, b$

$u=$ prescribed; otherwise $N_{x}=0$

$u=$ prescribed; otherwise $N_{x y}=0$

$v=$ prescribed; otherwise $N_{x y}=0$

$v=$ prescribed; otherwise $N_{y}=0$

$w=$ prescribed; otherwise $\frac{\partial M_{x}}{\partial_{x}}+\frac{\partial M_{x y}}{\partial y}=0$

$w=$ prescribed; otherwise $\frac{\partial M_{x y}}{\partial_{x}}+\frac{\partial M_{y}}{\partial_{y}}=0$

$\frac{\partial w}{\partial x}=$ prescribed; otherwise $M_{x}=0$

$\frac{\partial w}{\partial y}=$ prescribed; otherwise $M_{x y}=0$

$\varphi=$ prescribed; otherwise

$\alpha_{1} \frac{\partial S_{x}}{\partial_{x}}+\frac{\alpha_{1}+\alpha_{2}}{2} \frac{\partial S_{x y}}{\partial y}-\left(1+\alpha_{1}\right) Q_{x z}=0$

$\frac{\partial w}{\partial x}=$ prescribed; otherwise $M_{x y}=0$

$\frac{\partial w}{\partial y}=$ prescribed; otherwise $M_{y}=0$

$\varphi=$ prescribed; otherwise

$\frac{\partial \varphi}{\partial x}=$ prescribed; otherwise $\alpha_{1} S_{x}=0$

$\frac{\partial \varphi}{\partial y}=$ prescribed; otherwise $\frac{\alpha_{1}+\alpha_{2}}{2} S_{x y}=0$

\section{Closed-form solution}

The determination of displacements and stresses is of fundamental importance in the design of many structural components. The present boundary value problem associated with the equilibrium of laminated plates involves solving the operator equation, Eq. (11), subjected to the above given set of boundary conditions. An exact closed-form solution to Eq. (11) can be constructed

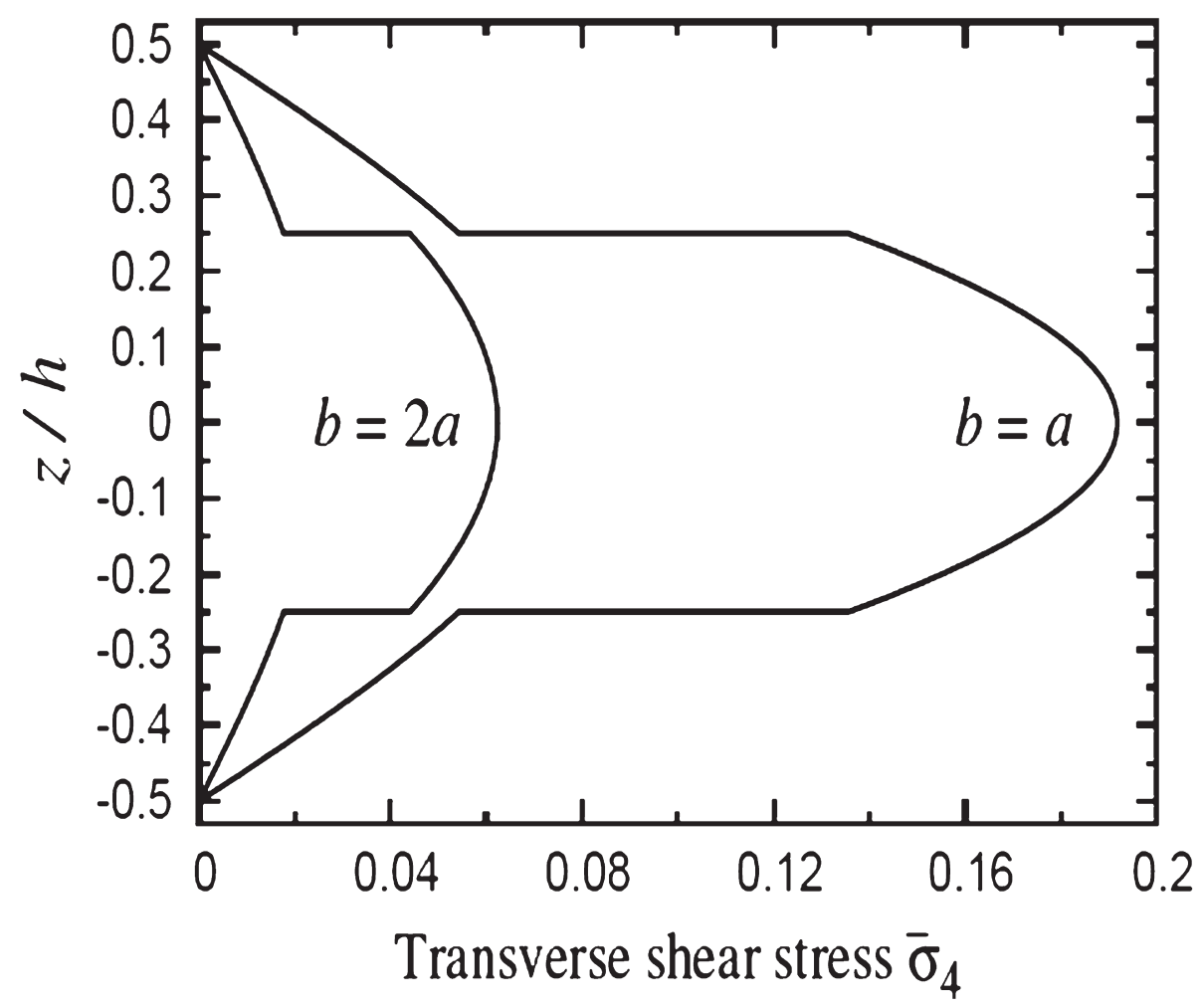

Figure 8. The transverse shear stress $\bar{\sigma}_{4}$ through-the-thickness of a $\left(0^{\circ} / 90^{\circ} / 90^{\circ} / 0^{\circ}\right)$ plate $(a / h=10)$. 
when the plate is of a rectangular geometry (figure 1) with the following edge conditions, loading and plate constructions.

The following set of simply supported boundary conditions along the edges of the plate is considered:

$$
\begin{aligned}
& v=w=\varphi=N_{1}=M_{1}=S_{1}=0 \quad \text { at } \quad x=0, a, \\
& u=w=\varphi=N_{2}=M_{2}=S_{2}=0 \text { at } \quad y=0, b \text {. }
\end{aligned}
$$

Under a general co-ordinate transformation, an initially orthotropic material becomes generally anisotropic. However, there are three specific coordinate transformations under which an orthotropic material retains monoclinic symmetry, namely, rotations about the axes $x, y$, or $z$. If the plate construction is cross-ply, the orthotropic material with respect to the old coordinate system follows under rotation through an angle $\theta_{k}\left(=0^{\circ}\right.$ or $\left.90^{\circ}\right)$ about the $z$-axis that the transformation formulae for the stiffnesses $c_{i j}^{(k)}$ are of the form (Bogdanovich \& Pastore 1996):

$$
\left\{\begin{array}{l}
c_{11} \\
c_{12} \\
c_{22} \\
c_{66}
\end{array}\right\}^{k}=\left[\begin{array}{llll}
c^{4} & 2 c^{2} s^{2} & s^{4} & 4 c^{2} s^{2} \\
c^{2} s^{2} & c^{4}+s^{4} & c^{2} s^{2} & -4 c^{2} s^{2} \\
s^{4} & 2 c^{2} s^{2} & c^{4} & 4 c^{2} s^{2} \\
c^{2} s^{2} & -2 c^{2} s^{2} & c^{2} s^{2} & \left(c^{2}-s^{2}\right)^{2}
\end{array}\right]\left\{\begin{array}{l}
c_{11} \\
c_{12} \\
c_{22} \\
c_{66}
\end{array}\right\}
$$

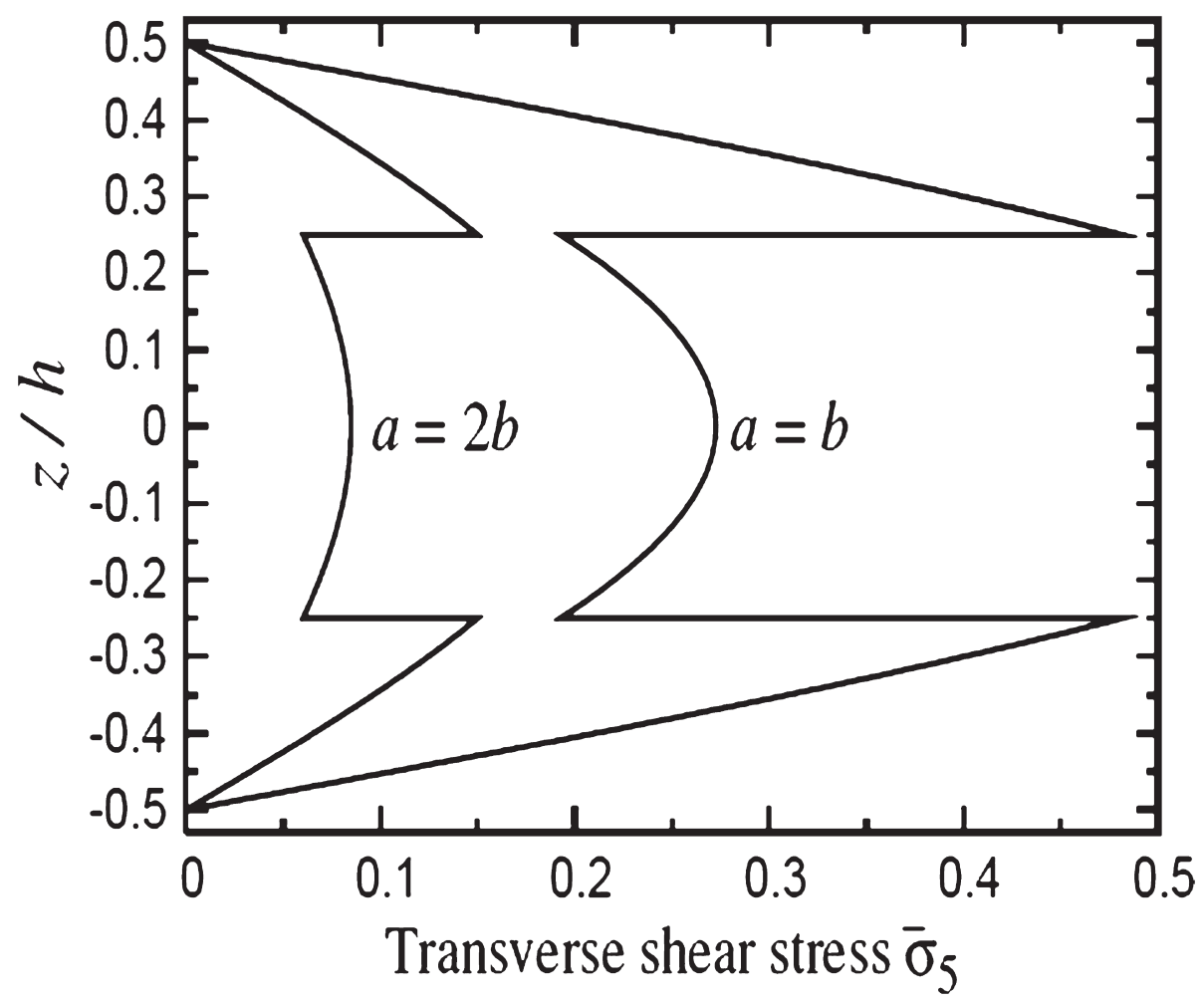

Figure 9. The transverse shear stress $\bar{\sigma}_{5}$ through-the-thickness of a $\left(0^{\circ} / 90^{\circ} / 90^{\circ} / 0^{\circ}\right)$ plate $(a / h=10)$. 


$$
c_{33}^{(k)}=c_{33}, \quad\left(\left\{\begin{array}{l}
c_{13} \\
c_{23}
\end{array}\right\}^{k},\left\{\begin{array}{l}
c_{44} \\
c_{55}
\end{array}\right\}^{k}\right)=\left[\begin{array}{ll}
c^{2} & s^{2} \\
s^{2} & c^{2}
\end{array}\right]\left(\left\{\begin{array}{l}
c_{13} \\
c_{23}
\end{array}\right\},\left\{\begin{array}{l}
c_{44} \\
c_{55}
\end{array}\right\}\right),
$$

where $c=\cos \theta_{k}, s=\sin \theta_{k}$ and $c_{i j}$ are the material stiffness of the lamina:

$$
\begin{gathered}
c_{11}=\frac{E_{1}\left(1-v_{23} v_{32}\right)}{\Delta}, c_{12}=\frac{E_{1}\left(v_{21}+v_{23} v_{31}\right)}{\Delta}=\frac{E_{2}\left(v_{12}+v_{32} v_{13}\right)}{\Delta}, c_{22}=\frac{E_{2}\left(1-v_{13} v_{31}\right)}{\Delta}, \\
c_{13}=\frac{E_{1}\left(v_{31}+v_{21} v_{32}\right)}{\Delta}=\frac{E_{3}\left(v_{13}+v_{12} v_{23}\right)}{\Delta}, c_{23}=\frac{E_{3}\left(v_{23}+v_{13} v_{21}\right)}{\Delta}=\frac{E_{2}\left(v_{32}+v_{12} v_{31}\right)}{\Delta}, \\
c_{33}=\frac{E_{3}\left(1-v_{12} v_{21}\right)}{\Delta}, \quad c_{44}=G_{23}, \quad c_{55}=G_{13}, \quad c_{66}=G_{12}, \\
\Delta=1=v_{12} v_{21}-v_{23} v_{32}-v_{31} v_{13}-2 v_{21} v_{32} v_{13},
\end{gathered}
$$

in which $E_{i}$ are Young's moduli in the material principal directions, $v_{i j}$ are Poisson's ratios, and $G_{i j}$ are shear moduli.

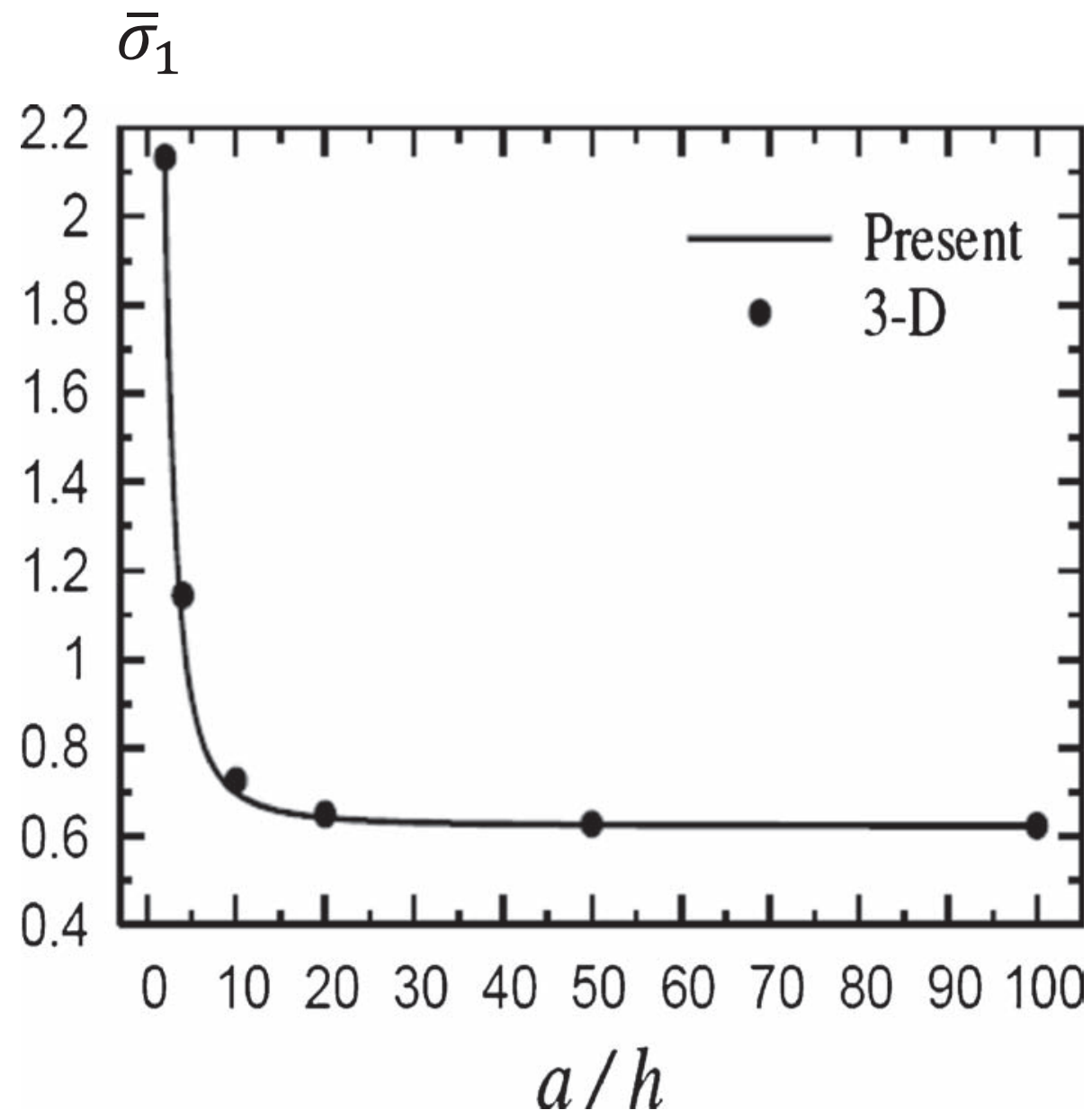

Figure 10. The in-plane longitudinal stress $\bar{\sigma}_{1}$ versus the side-to-thickness ratio $a / h$ of a $\left(0^{\circ} / 90^{\circ} / 90^{\circ}\right)$ rectangular plate $(b=3 a)$. 
To solve this problem, Navier presented the applied transverse sinusoidal load in the form:

$$
q=q_{0} \sin (\lambda x) \sin (\mu y),
$$

where $\lambda=\pi / a, \mu=\pi / b$, and $q_{0}$ is the intensity of the transverse load at the plate center. In addition, the displacements take the forms

$$
\left\{\begin{array}{c}
u \\
v \\
w \\
\varphi
\end{array}\right\}=\left\{\begin{array}{cc}
U \cos (\lambda x) & \sin (\mu y) \\
V \sin (\lambda x) & \cos (\mu y) \\
W \cos (\lambda x) & \sin (\mu y) \\
\Phi \sin (\lambda x) & \sin (\mu y)
\end{array}\right\},
$$

where $U, V, W$ and $\Phi$ are arbitrary parameters. The above solution form for $\{u, v, w, \varphi\}$ satisfies the simply supported boundary conditions and the parameters $U, V, W$, and $\Phi$ will be

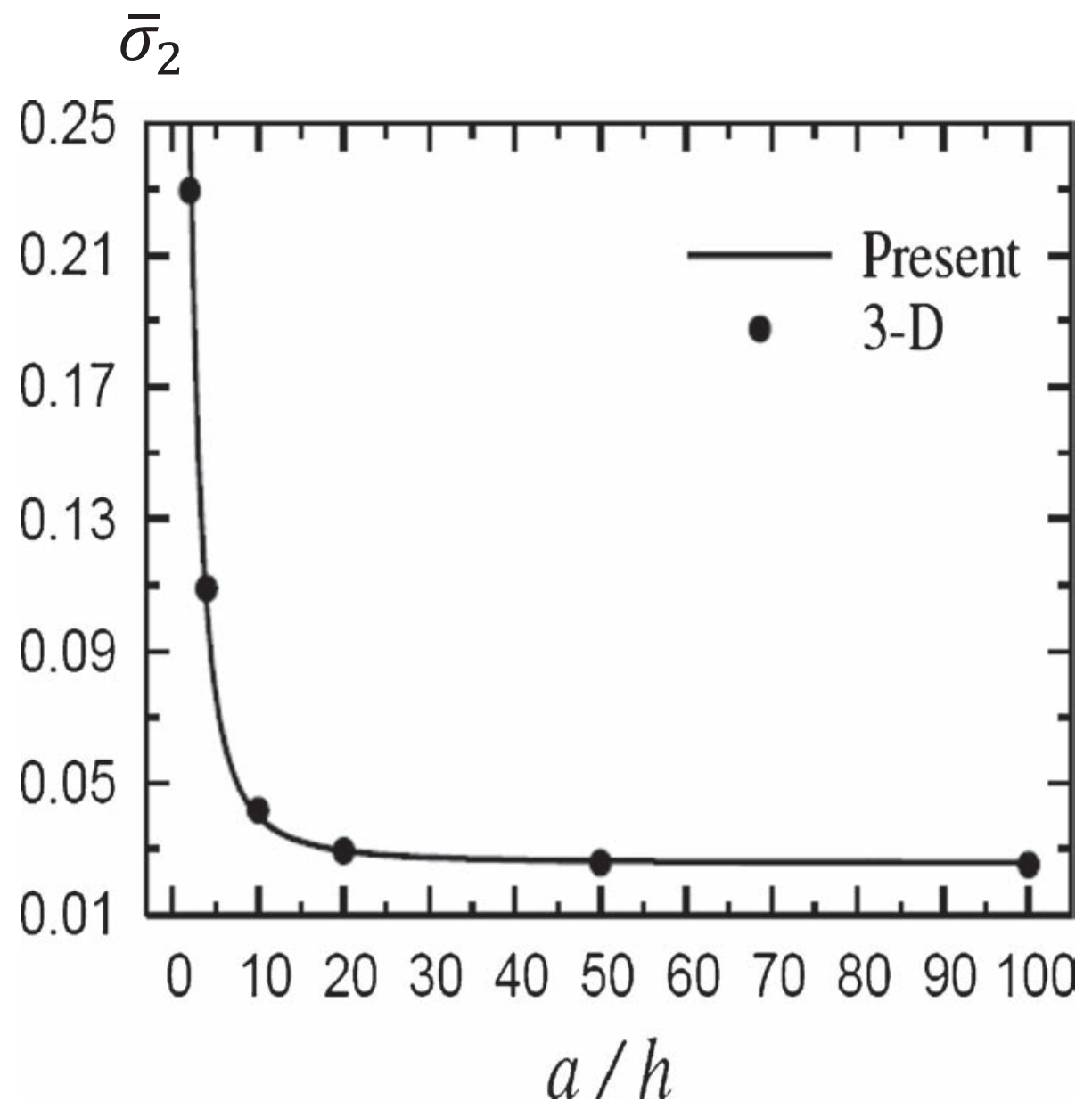

Figure 11. The in-plane normal stress $\bar{\sigma}_{2}$ versus the side-to-thickness ratio $a / h$ of a $\left(0^{\circ} / 90^{\circ} / 90^{\circ}\right)$ rectangular plate $(b=3 a)$. 
determined, subjected to the condition that the solution satisfies the differential equations given in Eq. (11). Substituting Eqs. (15) and (16) into Eq. (11), one obtains

$$
[\bar{J}]\{\Delta\}=\{\bar{Q}\},
$$

where $\{\Delta\}=\{U, V, W, \Phi\}^{t}$ and $\{\bar{Q}\}=\left\{0,0, q_{0}, 0\right\}^{t}$. Also, the elements of the symmetric matrix $[\bar{J}]$ are given in Appendix B.

\section{Numerical examples and discussion}

Numerical results for displacements and stresses are presented for symmetric cross-ply $\left(0^{\circ} / 90^{\circ} 90^{\circ} / 0^{\circ}\right)$ square and $\left(0^{\circ} / 90^{\circ} / 0^{\circ}\right)$ rectangular plates subjected to sinusoidal distributed loading. The results are compared with the most famous 3-D solution of Pagano (1970) and Pagano \& Hatfield (1972) (see also Zenkour (2007b)). The plate laminas are assumed to be of the

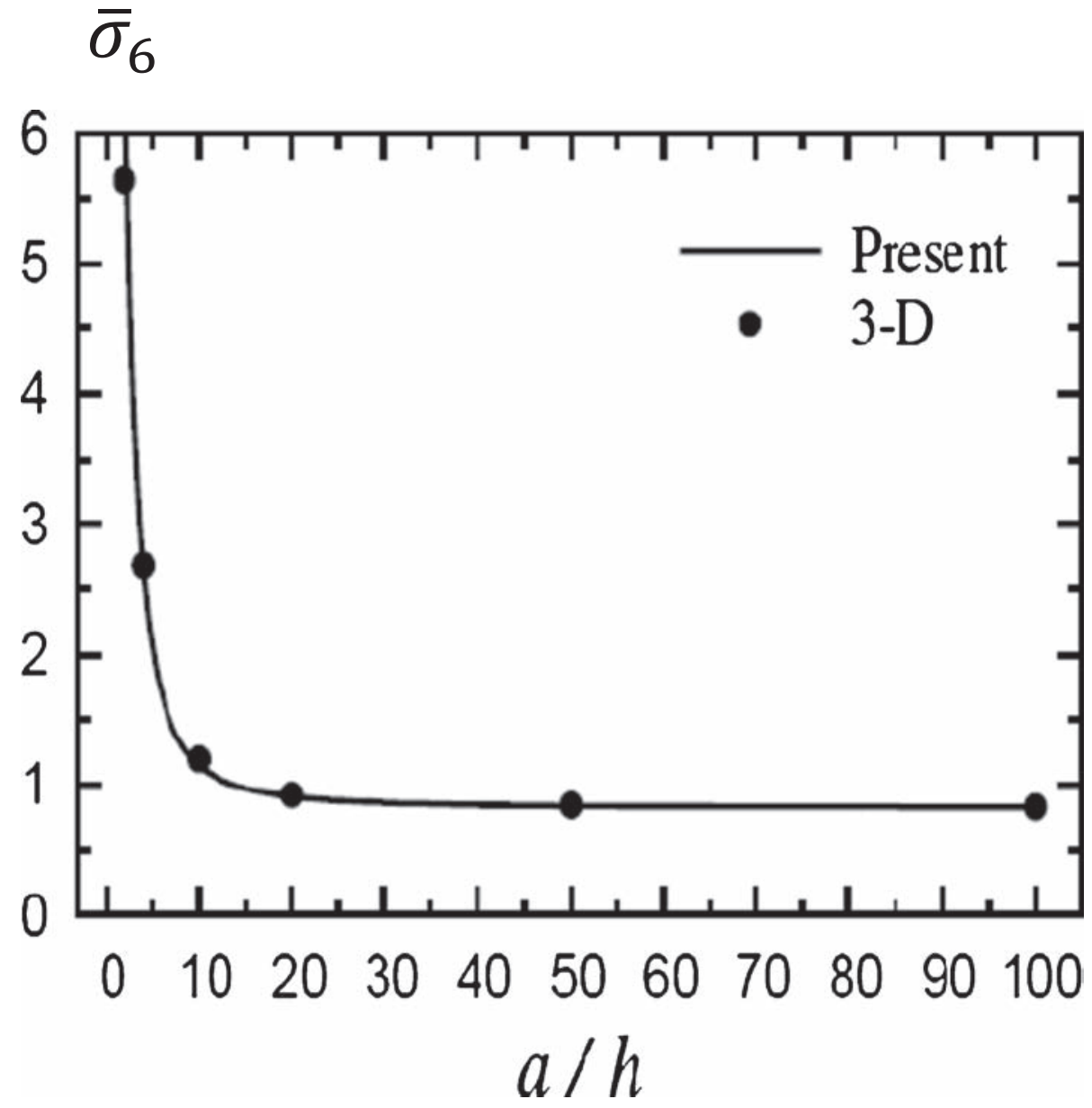

Figure 12. The in-plane tangential stress $\bar{\sigma}_{6}$ versus the side-to-thickness ratio $a / h$ of a $\left(0^{\circ} / 90^{\circ} / 90^{\circ}\right)$ rectangular plate $(b=3 a)$. 
same thickness and made of the same orthotropic material. The lamina properties are assumed to be:

$$
\begin{aligned}
& E_{1}=25 \mathrm{Msi}, \quad E_{2}=E_{3}=1 \mathrm{Msi}, \quad G_{12}=G_{13}=0.5 \mathrm{Msi}, \\
& G_{23}=0.2 \mathrm{Msi}, \quad v_{12}=v_{13}=v_{23}=0.25
\end{aligned}
$$

The dimensionless displacements and stresses are given by

$$
\begin{aligned}
& \bar{u}_{1}=-\frac{100 E_{3} h^{2}}{q_{0} a^{3}} u_{1}\left(0, \frac{b}{z}, \bar{z}\right), \quad \bar{u}_{2}=-\frac{100 E_{3} h^{2}}{q_{0} a^{3}} u_{2}\left(\frac{a}{2}, 0, \bar{z}\right), \quad \bar{u}_{3}=\frac{\pi^{4} Q h^{3}}{q_{0} a^{4}} u_{3}\left(\frac{a}{2}, \frac{b}{2}, \bar{z}\right), \\
& \bar{w}=\frac{100 E_{3} h^{3}}{q_{0} a^{4}} u_{3}\left(\frac{a}{2}, \frac{b}{2} \bar{z}\right), \quad \bar{\sigma}_{4}=\frac{h}{q_{0} a} \tau_{y z}\left(\frac{a}{2}, 0, \bar{z}\right), \quad \bar{\sigma}_{5}=\frac{h}{q_{0} a} \tau_{y z}\left(0, \frac{b}{2}, \bar{z}\right), \\
& \bar{\sigma}_{1}=\frac{h^{2}}{q_{0} a^{2}} \sigma_{x}\left(\frac{a}{2}, \frac{b}{2}, \bar{z}\right), \quad \bar{\sigma}_{2}=\frac{h^{2}}{q_{0} a^{2}} \sigma_{y}\left(\frac{a}{2}, \frac{b}{2}, \bar{z}\right), \quad \bar{\sigma}_{6}=-\frac{100 h^{2}}{q_{0} a^{2}} \tau_{x y}(0,0, \bar{z}),
\end{aligned}
$$

where

$$
\bar{z}=\frac{z}{h}, \quad Q=4 G_{12}+\frac{E_{1}+E_{2}\left(1+2 v_{23}\right)}{1-v_{12} v_{21}} .
$$

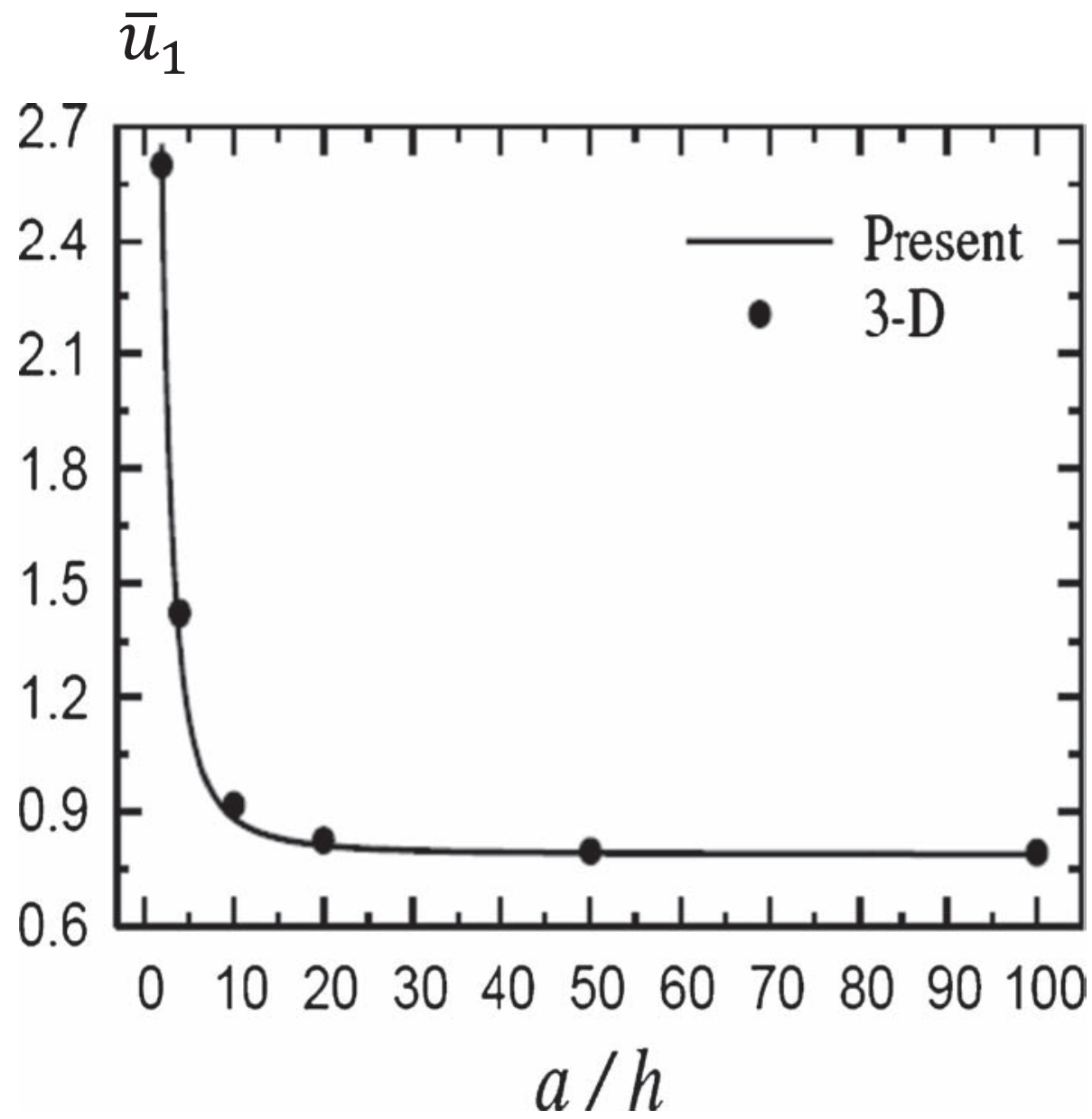

Figure 13. The in-plane displacement $\bar{u}_{1}$ versus the side-to-thickness ratio $a / h$ of a $\left(0^{\circ} / 90^{\circ} / 90^{\circ}\right)$ rectangular plate $(b=3 a)$. 
It is found that the two parameters $\alpha_{1}$ and $\alpha_{2}$ should be given in terms of the side-to-thickness ratios as

$$
\alpha_{1}=\frac{50 b_{2}}{h^{2}\left(\frac{b}{h}+\frac{1}{4}\right)}, \quad \alpha_{2}=\frac{50 a^{2}}{h^{2}\left(\frac{115}{100} \sqrt{\left(\frac{a}{h}\right)^{3}}+\frac{1}{4}\right)} .
$$

The above expressions for $\alpha_{1}$ and $\alpha_{2}$ give accurate results when compared the present model with the 3-D one. Note that, the quantities $\bar{\sigma}_{1}, \bar{\sigma}_{6}, \bar{u}_{1}$, and $\bar{u}_{2}$ are computed at $\bar{z}=1 / 2$ while the normal displacements $\bar{w}$ and $\bar{u}_{3}$ are computed at $\bar{z}=1 / 6$. In addition, the in-plane normal stress $\bar{\sigma}_{2}$ is computed in the $\left(0^{\circ} / 90^{\circ} / 90^{\circ} / 0^{\circ}\right)$ square plate at $\bar{z}=1 / 4$ while it is computed in the $\left(0^{\circ} / 90^{\circ} / 0^{\circ}\right)$ rectangular plate at $\bar{z}=1 / 6$. Dimensionless displacements of and stresses in symmetric $\left(0^{\circ} / 90^{\circ} / 90^{\circ} / 0^{\circ}\right)$ square plates are presented in figures 2-9. All stresses and displacements are compared well with the corresponding 3-D solutions (Pagano 1970; Pagano \& Hatfield 1972). Low sensitivity relative errors occur only at $a / h=2$. In addition, the transverse shear stresses are plotted through-the-thickness of the $\left(0^{\circ} / 90^{\circ} / 90^{\circ} / 0^{\circ}\right)$ square and rectangular

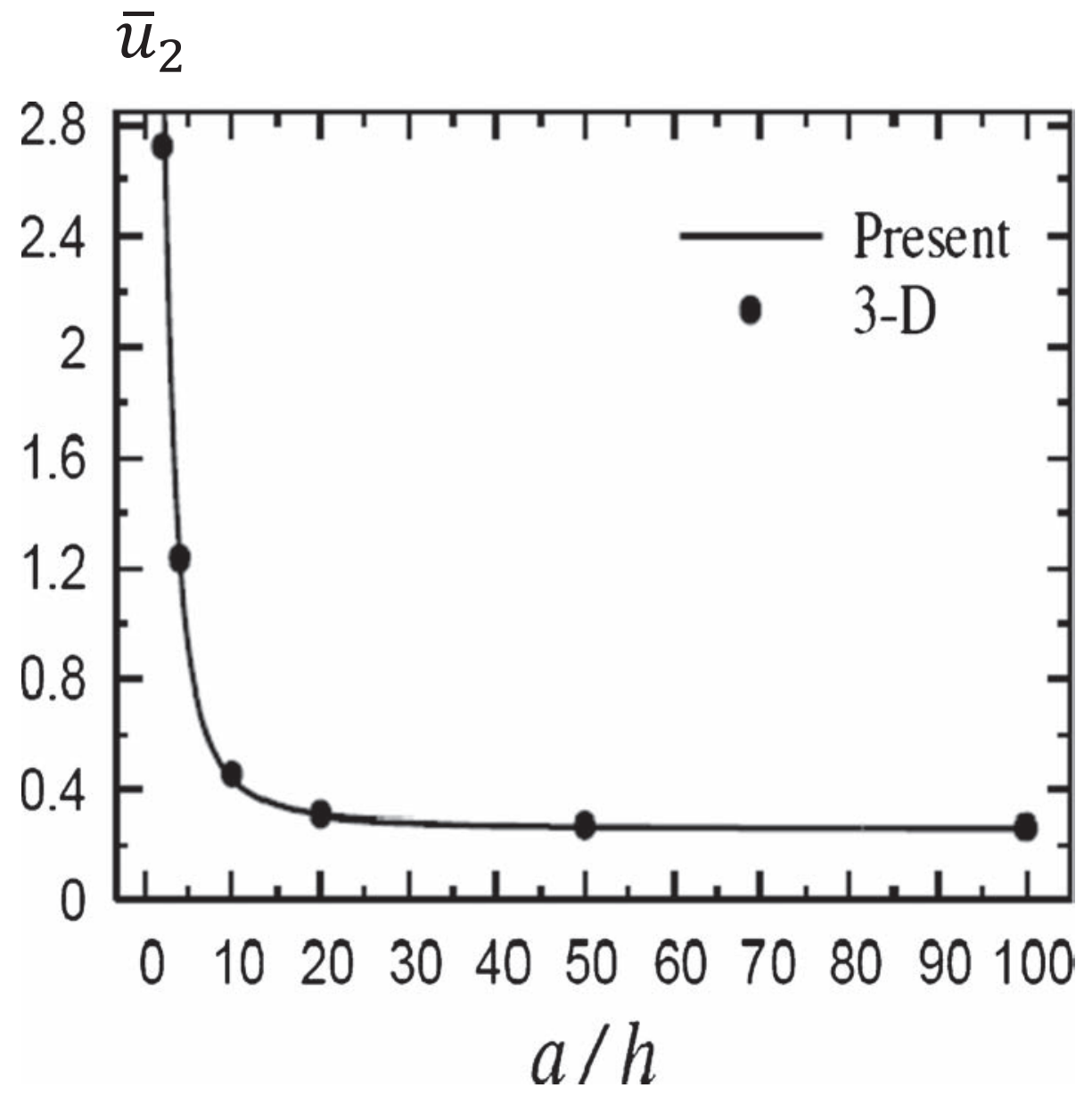

Figure 14. The in-plane displacement $\bar{u}_{2}$ versus the side-to-thickness ratio $a / h$ of a $\left(0^{\circ} / 90^{\circ} / 90^{\circ}\right)$ rectangular plate $(b=3 a)$. 


\section{$\bar{W}$}

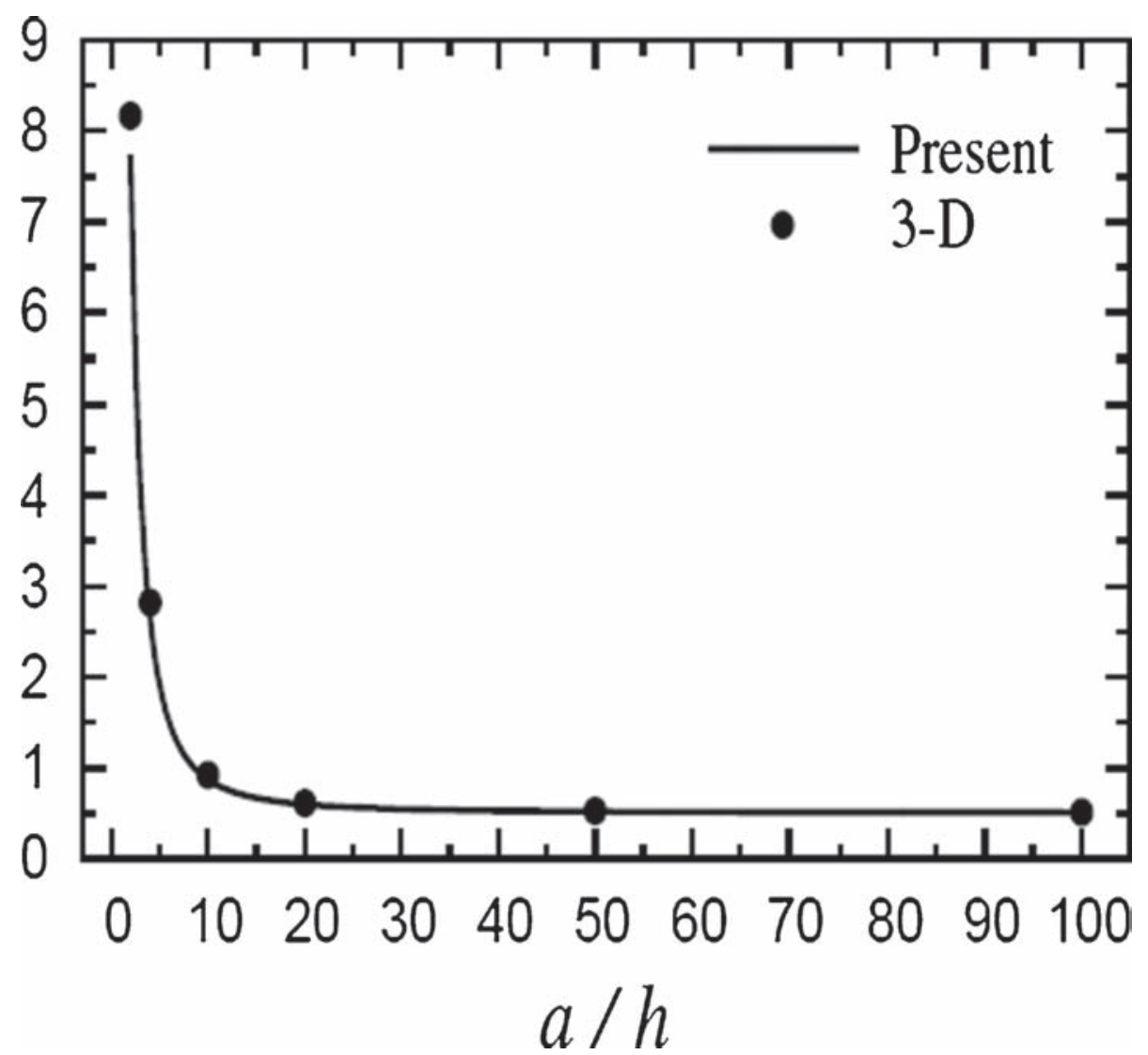

Figure 15. The out-of-plane displacement $\bar{w}$ versus the side-to-thickness ratio $a / h$ of a $\left(0^{\circ} / 90^{\circ} / 90^{\circ}\right)$ rectangular plate $(b=3 a)$.

plates. For the sake of completeness and discussion, the stresses in and displacements of symmetric $\left(0^{\circ} / 90^{\circ} / 0^{\circ}\right)$ rectangular plates are also presented in figures $10-15$. The results are more accurate and compared well with the 3-D solution.

\section{Conclusions}

The explicit governing equations of a simplified 4-unkoun sinusoidal shear and normal deformations plate theory are presented for the cross-ply laminated plates. This paper addresses the bending analysis and accounts for the through-the-thickness deformations. Numerical results demonstrate the efficiency of the present approach. The displacement field of the classical thin plate theory (CPT) is obtained easily from Eq. (3) by setting $f(z)=0$. However, the displacement field of the first-order shear deformation plate theory (FPT) is obtained by setting

$$
f(z)=z, \quad w \rightarrow w-\varphi, \quad \psi_{1}=\left(\alpha_{1}+1\right) \frac{\partial \varphi}{\partial x}, \quad \psi_{2}=\left(\alpha_{2}+1\right) \frac{\partial \varphi}{\partial y} .
$$


We found that the two parameters $\alpha_{1}$ and $\alpha_{2}$ should not be equal. The author is looking forward to that all interested researchers could generate other forms of the coefficients $\alpha_{1}$ and $\alpha_{2}$ in order to get results with high accuracy. An extension to the present simplified 4-uknwon theory will be presented for the bending of functionally graded plates in future works.

\section{Appendix A}

The elements $J_{i j}=J_{j i}$ of the matrix elements [ $\left.J\right]$ appeared in Eq. (11) are given by

$$
\begin{aligned}
J_{11}= & A_{11} d_{11}+A_{66} d_{22}, \quad J_{12}=\left(A_{12}+A_{66}\right) d_{12}, \\
J_{13}= & -B_{11} d_{111}-\left(B_{12}+2 B_{66}\right) d_{122}, \\
J_{14}= & \alpha_{3} G_{13}^{a} d_{1}+\alpha_{1} B_{11}^{a} d_{111}+\left[\alpha_{2} B_{12}^{a}+\left(\alpha_{1}+\alpha_{2}\right) B_{66}^{a}\right] d_{122}, \\
J_{22}= & A_{66} d_{11}+A_{22} d_{22}, \quad J_{23}=-\left(B_{12}+2 B_{66}\right) d_{122}-B_{22} d_{222}, \\
J_{24}= & \alpha_{3} G_{23}^{a} d_{2}+\alpha_{2} B_{22}^{a} d_{222}+\left[\alpha_{1} B_{12}^{a}+\left(\alpha_{1}+\alpha_{2}\right) B_{66}^{a}\right] d_{122}, \\
J_{33}= & -D_{11} d_{1111}-2\left(D_{12}+2 D_{66}\right) d_{1122}-D_{22} d_{2222}, \\
J_{34}= & \alpha_{3}\left(H_{23}^{a} d_{11}+H_{23}^{a} d_{22}\right)+\alpha_{1} D_{11}^{a} d_{1111}+\left(\alpha_{1}+\alpha_{2}\right)\left[D_{12}^{a}+2 D_{66}^{a}\right] d_{1122} \\
& +\alpha_{2} D_{22}^{a} d_{2222}, \\
J_{44}= & \alpha_{3}^{2} P_{33}^{a}+\left[2 \alpha_{1} \alpha_{3} L_{13}^{a}-\left(\alpha_{1}+\alpha_{3}\right)^{2} A_{55}^{a}\right] d_{11}+\left[2 \alpha_{2} \alpha_{3} L_{23}^{a}-\left(\alpha_{2}+\alpha_{3}\right)^{2} A_{44}^{a}\right] d_{22} \\
& +\alpha_{1}^{2} F_{11}^{2} d_{1111}+\left[2 \alpha_{1} \alpha_{2} F_{12}^{a}+\left(\alpha_{1}+\alpha_{2}\right)^{2} F_{66}^{a}\right] d_{1122}+\alpha_{2}^{2} F_{22}^{a} d_{2222},
\end{aligned}
$$

where $d_{i}, d_{i j}, d_{i j l}, d_{i j l m}$ are the following differential operators:

$$
d_{i}=\frac{\partial}{\partial_{x_{i}}}, \quad d_{i j}=\frac{\partial^{2}}{\partial_{x_{i}} \partial_{x_{j}}}, \quad d_{i j l}=\frac{\partial^{3}}{\partial_{x_{i}} \partial_{x_{j}} \partial_{x_{l}}}, \quad d_{i j l m}=\frac{\partial^{4}}{\partial_{x_{i}} \partial_{x_{j}} \partial_{x_{l}} \partial_{x_{m}}} .
$$

\section{Appendix B}

The elements $\bar{J}_{i j}=\bar{J}_{i j}$ of the matrix elements $[\bar{J}]$ appeared in Eq. (17) are given by

$$
\begin{aligned}
\bar{J}_{11}= & A_{11} \lambda^{2}+A_{66} \mu^{2}, \quad \bar{J}_{12}=\left(A_{12}+A_{66}\right) \lambda \mu, \\
\bar{J}_{13}= & -\lambda\left[B_{11} \lambda^{2}+\left(B_{12}+2 B_{66}\right) \mu^{2}\right], \\
\bar{J}_{14}= & \lambda\left\{\alpha_{1} B_{11}^{a} \lambda^{2}+\left[\alpha_{2} B_{12}^{a}+\left(\alpha_{1}+\alpha_{2}\right) B_{66}^{a}\right] \mu^{2}-\alpha_{3} G_{13}^{a}\right\}, \\
\bar{J}_{22}= & A_{66} \lambda^{2}+A_{22} \mu^{2}, \quad \bar{J}_{23}=-\mu\left[\left(B_{12}+2 B_{66}\right) \lambda^{2}+B_{22} \mu^{2}\right], \\
\bar{J}_{24}= & \mu\left\{\alpha_{2} B_{22}^{a} \mu^{2}+\left[\alpha_{1} B_{12}^{a}+\left(\alpha_{1}+\alpha_{2}\right) B_{66}^{a}\right] \lambda^{2}-\alpha_{3} G_{23}^{a}\right\}, \\
\bar{J}_{33}= & D_{11} \lambda^{4}+2\left(D_{12}+2 D_{66}\right) \lambda^{2} \mu^{2}+D_{22} \mu^{4}, \\
\bar{J}_{34}= & \alpha_{3}\left(H_{13}^{a} \lambda^{2}+H_{23}^{a} \mu^{2}\right)-\alpha_{1} D_{11}^{a} \lambda^{4}-\left(\alpha_{1}+\alpha_{2}\right)\left[D_{12}^{a}+2 D_{66}^{a}\right] \lambda^{2} \mu^{2}-\alpha_{2} D_{22}^{a} \mu^{4}, \\
\bar{J}_{44}= & \alpha_{3}^{2} P_{33}^{a}-\left[2 \alpha_{1} \alpha_{3} L_{13}^{a}-\left(\alpha_{1}+\alpha_{3}\right)^{2} A_{55}^{a}\right] \lambda^{2}-\left[2 \alpha_{2} \alpha_{3} L_{23}^{a}-\left(\alpha_{2}+\alpha_{3}\right)^{2} A_{44}^{a}\right] \mu^{2} \\
& +\alpha_{1}^{2} F_{11}^{a} \lambda^{4}+\left[2 \alpha_{1} \alpha_{2} F_{12}^{a}+\left(\alpha_{1}+\alpha_{2}\right)^{2} F_{66}^{a}\right] \lambda^{2} \mu^{2}+\alpha_{2}^{2} F_{22}^{a} \mu^{4} .
\end{aligned}
$$




\section{References}

Aydogdu M 2009 A new shear deformation theory for laminated composite plate. Compos. Struct. 89: 94-101

Batista M 2010 The derivation of the equations of moderately thick plates by the method of successive approximations. Acta Mech. 210: 159-168

Bogdanovich A E and Pastore C M 1996 Mechanics of textile and laminated composites with applications to structural analysis. London: Chapman \& Hall

Ferreira A J M and Roque C M C 2011 Analysis of thick plates by radial basis functions. Acta Mech 217: $177-190$

Ferreira A J M, Roque C M C and Jorge R M N 2005 Analysis of composite plates by trigonometric shear deformation theory and multiquadrics. Comput. Struct. 83: 2225-2237

Fiedler L, Lacarbonara W and Vestroni F 2010 A generalized higher-order theory for multi-layered, sheardeformable composite plates. Acta Mech. 209: 85-98

Jun L and Hongxing H 2009 Dynamic stiffness analysis of laminated composite beams using trigonometric shear deformation theory. Compos. Struct. 89: 433-442

Kant T, Gupta A B, Pendhari S S and Desai Y M 2008 Elasticity solution for cross-ply composite and sandwich laminates. Compos. Struct. 83: 13-24

Kapuria S and Kumari P 2010 Three-dimensional piezoelasticity solution for dynamics of cross-ply cylindrical shells integrated with piezoelectric fiber reinforced composite actuators and sensors. Compos. Struct. 92: 2431-2444

Khabbaz R S, Manshadi B D and Abedian A 2009 Nonlinear analysis of FGM plates under pressure loads using the higher-order shear deformation theories. Compos. Struct. 89: 333-344

Love A E H 1944 A treatise on the mathematical theory of elasticity. 4th ed. New York, USA: Dover Publ

Mantari J L, Oktem A S and Soares C G 2012a Bending response of functionally graded plates by using a new higher order shear deformation theory. Compos. Struct. 94: 714-723

Mantari J L, Oktem A S and Soares C G 2012b A new higher order shear deformation theory for sandwich and composite laminated plates. Compos. B 43: 1489-1499

Mindlin R D 1951 Influence of rotatory inertia and shear on flexural motions of isotropic, elastic plates. ASME. J. Appl. Mech. 18: 31-38

Neves A M A, Ferreira A J M, Carrera E, Cinefra M, Roque C M C, Jorge R M N and Soares C M M A quasi-3D hyperbolic shear deformation theory for the static and free vibration analysis of functionally graded plates. Compos. Struct. 94: 1814-1825

Neves A M A, Ferreira A J M, Carrera E, Roque C M C, Cinefra M, Jorge R M N and Soares C M M 2012b A quasi-3D sinusoidal shear deformation theory for the static and free vibration analysis of functionally graded plates. Compos. B 43: 711-725

Pagano N J 1970 Exact solutions for rectangular bidirectional composites and sandwich plates. J. Compos. Mater. 4: 20-24

Pagano N J and Hatfield S J 1972 Elastic behaviour of multilayered bidirectional composites. AIAA J. 10: 931-933

Reddy J N 1984 A simple higher-order theory for laminated composite plates. J. Appl. Mech. 51: 745-752

Reissner E 1944 On the theory of bending of elastic plates. J. Math Phys. 23: 184-191

Reissner E 1945 The effect of transverse shear deformation on the bending of elastic plates. ASME. J. Appl. Mech. 12: 69-77

Touratier M 1991 An efficient standard plate theory. Int. J. Eng. Sci. 29: 901-916

Xiang S, Wang K, Ai Y, Sha Y and Shi H 2009 Analysis of isotropic, sandwich and laminated plates by a meshless method and various shear deformation theories. Compos. Struct. 91: 31-37

Zenkour A M 2004a Analytical solution for bending of cross-ply laminated plates under thermo-mechanical loading. Compos. Struct. 65: 367-379

Zenkour A M 2004b Thermal effects on the bending response of fiber-reinforced viscoelastic composite plates using a sinusoidal shear deformation theory. Acta Mech. 171: 171-187 
Zenkour A M 2005a A comprehensive analysis of functionally graded sandwich plates: Part 1 Deflection and stresses, Part 2 Buckling and free vibration. Int. J. Solids Struct. 42: 5224-5258

Zenkour A M 2005b On vibration of functionally graded plates according to a refined trigonometric plate theory. Int. J. Struct. Stab. Dynam. 5: 279-297

Zenkour A M 2006 Generalized shear deformation theory for bending analysis of functionally graded plates. Appl. Math. Model 30: 67-84

Zenkour A M 2007a Benchmark trigonometric and 3-D elasticity solutions for an exponentially graded thick rectangular plate. Arch. Appl. Mech. 77: 197-214

Zenkour A M 2007b Three-dimensional elasticity solution for uniformly loaded cross-ply laminates and sandwich plates. J. Sandw. Struct. Mater. 9: 213-238 